\title{
UAV Trajectory Design and Bandwidth Allocation for Coverage Maximization with Energy and Time Constraints
}

\author{
Xiaoye Jing and Christos Masouros \\ Department of Electronic and Electrical Engineering \\ University College London \\ London, UK \\ x.jing@ucl.ac.uk, chris.masouros@ieee.org
}

\begin{abstract}
Unmanned Aerial Vehicle (UAV) networks have recently gained interest, owing to the mobility of UAVs that can be exploited to improve channel conditions and user coverage. In this paper, we consider a scenario where a rotary-wing UAV is dispatched for covering a maximum number of ground users by jointly optimizing the UAV trajectory and bandwidth allocation, under constraints of pre-determined maximal total flight time and on-board energy. The problem is difficult to solve since has nonconvex constraints and includes infinite variables over time. As such, we propose an iterative algorithm with guaranteed convergence by applying block coordinate descent and successive convex approximation techniques. We further exploit the path discretization to formulate the original problem into an optimization formulation with finite variables. We deploy a UAV circular trajectory as the benchmark. The numerical results show that the proposed algorithm significantly outperforms the benchmark scheme and the bandwidth allocation can improve UAV coverage compared with the UAV trajectory only with time partitioning.
\end{abstract}

Index Terms-UAV communication, trajectory design, bandwidth allocation.

\section{INTRODUCTION}

Unmanned aerial vehicles (UAVs) provide wireless communication solutions in many real-world scenarios and thus gaining significant popularity in research [1], [2]. In one of their key use cases, UAVs can be applied as aerial base stations (BSs) and deployed in crowded areas to ease the burden of existing cellular systems [3], [4]. Moreover, the deployment of UAVs is also relevant in emergency or disaster scenarios where ground communication infrastructures are damaged [2], [5].

The authors in [6] studied the trade-off between altitude of static UAV and its coverage area. Algorithms were proposed to maximize coverage by multi-static UAVs deployment [7]-[10]. The work in [7] considered the effect of inter-cell interference. A research that focused on the coverage-efficient and energyefficient deployment of static UAVs by leveraging geometrical

\footnotetext{
This project has received funding from the European Union's Horizon 2020 research and innovation programme under the Marie Skłodowska-Curie grant agreement No 812991.
}

978-1-7281-4490-0/20/\$31.00 @ 2020 IEEE relaxation and clustering methods has been proposed [10], [11]. By exploiting the high mobility of UAVs, the communication path loss can be decreased due to the reduced distance between a UAV and users. Therefore, recent study has shifted from static UAVs to moving UAVs [12]. A cyclical multiple access (CMA) method of UAV communication based on communication delay-constrained were proposed in [13] and [14]. Since the UAV has limited on-board energy, recent studies considered energy constraints in UAV communication optimization, which included energy modelling [15], power minimization [16] and transmission delay minimization [17].

In particular, closest to the scope of our study, in [17], the objective was to minimize flight duration of the UAV with an aim of minimizing the communication delay of ground users (GUs). Meanwhile, UAV communication with bandwidth allocation was studied to improve communication performance. In most studies, a time discretization was exploited to illustrate UAV trajectory and ignored that variable time slot in a trajectory design can effect UAV communication performance.

In this paper, we consider a scenario that a UAV flies to serve GUs. The UAV starts from a charging base station (CBS) and is required to fly back to this CBS for recharging before exhausting its energy within allowed time period. The above two conditions necessitate both a maximal total flight time and a total energy constraint in our trajectory design. We assume that a user is covered only when its entire data request is delivered. Our aim is to maximize the number of covered users by jointly optimizing the UAV trajectory, user scheduling and bandwidth allocation. However, such a joint optimization problem is not only nonconvex but also contains infinite variables, and is thus challenging to solve. Therefore, an iterative algorithm based on successive convex approximation (SCA) and block coordinate descent (BCD) is exploited for this specific problem. Path discretization is used to transfer the infinite variable into a discrete form [18].

\section{Problem Formulation}

As shown in Fig. 1, we consider a square target area of dimension $L_{s}$ by $L_{s}$ containing $M$ delay-constrained GUs with low mobility noted by dots. The $m$-th user position is described 
as $\mathbf{w}_{m}=\left[x_{m}, y_{m}\right] \in \mathbb{R}^{1 \times 2}$. The UAV is dispatched from CBS (the square in Fig. 1) and tries to cover as many users as possible within the pre-determined time period and energy, and finally flies back to CBS.

Since time is an optimization variable, the formulation has infinite variables. To solve this problem, we discretize original optimization variables into finite variables. Since the universal time discretization requires flying time to be discretized into $N$ fixed time slots, it is not suitable for the slot time optimization in our paper. Therefore, we exploit another discretization method called path diescretization [18], to illustrate the UAV trajectory in our formulation. We assume that the UAV is flying with a fixed altitude $H$. The UAV total path is discretized into $N$ line segments which are denoted by $N+1$ locations, $\mathbf{s}[n]=\left[s_{x}[n], s_{y}[n]\right] \in \mathbb{R}^{1 \times 2}, n \in N$. The length of path slot $n$ is $\Delta_{n}$. The UAV flying duration in path slot $n$ is $T[n]$. The first and final locations of UAV path are both CBS. We assume that within each path slot, the UAV flies with a constant velocity, where $\mathbf{v}[n]=(\mathbf{s}[n+1]-\mathbf{s}[n]) / T[n]$ and that $\Delta_{n}$ should be chosen with constraints

$$
\Delta_{n}=\|\mathbf{s}[n+1]-\mathbf{s}[n]\| \leq \Delta_{\max }, \forall n
$$

For simplicity [18]-[20], we assume that UAV air-to-ground (AtG) links are dominated by Light of Sight (LoS) channels. Correspondingly, the path-varying channel for user $m$ at path slot $n$ is expressed as

$$
h_{m}[n]=\frac{\beta_{0}}{d_{m}[n]^{2}}=\frac{\beta_{0}}{H^{2}+\left\|\mathbf{s}[n]-\mathbf{w}_{m}\right\|^{2}}
$$

where we denote $\beta_{0}$ the channel power at the reference distance $d=1 \mathrm{~m}$ and denote $d_{m}[n]$ the distance from the UAV to user $m$ at path slot $n$.

In each path slot, the total bandwidth for communication is $B$. We employ frequency division multiple access (FDMA) scheme for bandwidth allocation to all users. Specifically, at path slot $n$, we denote $\alpha_{m n}$ as the fraction of $B$ that UAV uses to communicate with user $m$. We then have the constraint

$$
\sum_{m=1}^{M} \alpha_{m n} \leq 1, \forall n
$$

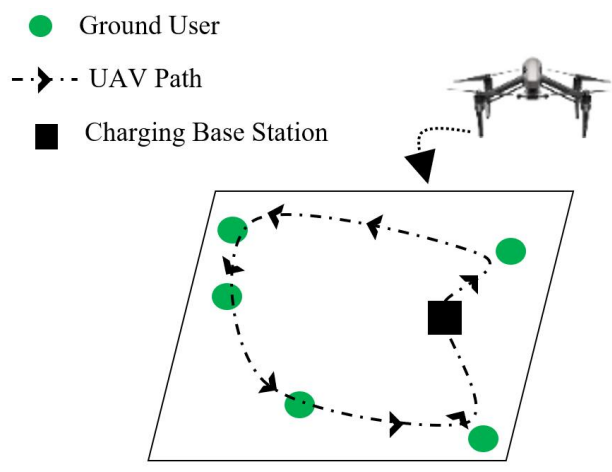

Fig. 1. The rotary-wing UAV serving users
Meanwhile, at each path slot, the maximal time for the UAV to send data for a user is $T[n]$. We define a variable $t_{m n}$ indicating the allocated time to communicate with aerial base station for the user $m$ at path slot $n$, where $t_{m n} \leq T[n]$.

That means the UAV can connect user $m$ at path slot $n$ with allocated bandwidth $\alpha_{m n} B$ and allocated time $t_{m n}$. The aggregated communication data bits between user $m$ and the $\mathrm{UAV}$ at path slot $n$ is expressed as

$$
b_{m}[n]=\alpha_{m n} t_{m n} B \log _{2}\left(1+\frac{P h_{m}[n]}{\alpha_{m n} B \sigma^{2}}\right)
$$

where $P$ and $\sigma^{2}$ denote the UAV transmit power and the noise power spectral density in watts/Hz respectively. Finally, the achievable total data for user $m$ in the unit of bits during whole flying period is

$$
b_{m}=\sum_{n=1}^{N} \alpha_{m n} t_{m n} B \log _{2}\left(1+\frac{\gamma_{m}[n]}{\alpha_{m n}}\right)
$$

where denotes $\gamma_{m}[n]=P \zeta_{0} /\left(H^{2}+\left\|\mathbf{s}[n]-\mathbf{w}_{m}\right\|^{2}\right)$ and denotes $\zeta_{0}=\beta_{0} /\left(B \sigma^{2}\right)$.

The total power consumption of a UAV consists the power consumed for communication and for supporting its mobility. The communication-related power is denoted by $P$ which is a constant in this paper. The propulsion energy depends on the UAV flying speed and acceleration. We ignore the additional energy consumed by UAV acceleration for a more tractable optimization [18]. The power about communication is ignored here since it is much lower than propulsion power. Thus the power consumption can be modeled as

$$
\begin{aligned}
P(V) & =P_{0}\left(1+\frac{3 V^{2}}{U_{\mathrm{tip}}^{2}}\right) \\
& +P_{\mathrm{i}}\left(\sqrt{\left(1+\frac{V^{4}}{4 v_{0}}\right)}-\frac{V^{2}}{2 v_{0}^{2}}\right)^{1 / 2}+\frac{1}{2} d_{0} \rho s A V^{3}
\end{aligned}
$$

where $P_{0}$ and $P_{\mathrm{i}}$ are the blade profile power and induced power in hovering status; $U_{\text {tip }}$ is the tip speed of the rotor blade; $v_{0}$ is the mean rotor induced velocity in forward flying; $d_{0}$ is the Fuselage drag ratio; $\rho$ is the air density; $s$ is rotor solidity and $A$ denotes rotor disc area. The energy consumption function parameters are shown in Table I.

Our aim is to maximize the number of covered users with time and energy constraints. We use a binary variable $\rho_{m}$ to denote the cover status of user $m$. We assume $Q_{m}$ as the data requested by user $m$. If the received data of user $m$ is no less than $Q_{m}, \rho_{m}=1$, and otherwise $\rho_{m}=0$. We

TABLE I

ENERGY CONSUMPTION FUNCTION PARAMETERS

\begin{tabular}{|c|c|c|c|}
\hline parameter & value & parameter & value \\
\hline$P_{0}$ & $17.6 \mathrm{~W}$ & $P_{\mathrm{i}}$ & $-43.8 \mathrm{~W}$ \\
\hline$U_{\mathrm{tip}}$ & $80 \mathrm{~m} / \mathrm{s}$ & $v_{0}$ & $3.19 \mathrm{~m} / \mathrm{s}$ \\
\hline$d_{0}$ & 0.81 & $s$ & $0.037 \mathrm{~m}^{3}$ \\
\hline$\rho$ & $1.23 \mathrm{~kg} / \mathrm{m}^{3}$ & $A$ & $0.5 \mathrm{~m}^{2}$ \\
\hline
\end{tabular}


denote $T_{\text {tot }}$ and $E_{\text {tot }}$ as the pre-determined maximal flying time and the on-board energy of UAV respectively. $\mathbf{s}_{0}$ is CBS. $V_{\max }$ is the UAV maximum allowed speed. In addition, we use $\Delta_{n} / T[n]$ to replace speed in energy consumption function (6). The optimization problem is then formulated as

$$
\begin{aligned}
(\mathrm{P} 1): & \max _{\left\{t_{m n}, \mathbf{s}[n], T[n], \alpha_{m n}, \rho_{m}\right\}} \sum_{m=1}^{M} \rho_{m} \\
\text { s.t. } & \sum_{n=1}^{N} \alpha_{m n} t_{m n} B \log _{2}\left(1+\frac{\gamma_{m}[n]}{\alpha_{m n}}\right) \geq \rho_{m} Q_{m}, \forall m \\
& \rho_{m} \in\{0,1\}, \forall m \\
& \mathbf{s}[1]=\mathbf{s}_{0}, \mathbf{s}[N+1]=\mathbf{s}_{0} \\
& \|\mathbf{s}[n+1]-\mathbf{s}[n]\| \leq \Delta_{\max }, \forall n \\
& \|\mathbf{s}[n+1]-\mathbf{s}[n]\| \leq V_{\max } T[n], \forall n \\
& 0 \leq t_{m n} \leq T[n], \forall m, n, \sum_{n=1}^{N} T[n] \leq T_{\mathrm{tot}} \\
& \alpha_{m n} \geq 0, \forall m, n, \quad \sum_{m=1}^{M} \alpha_{m n} \leq 1, \forall n \\
& P_{0} \sum_{n=1}^{N}\left(T[n]+\frac{3 \Delta_{n}^{2}}{U_{\mathrm{tip}}^{2} T[n]}\right) \\
& +P_{\mathrm{i}} \sum_{n=1}^{N}\left(\sqrt{\left(T[n]^{4}+\frac{\Delta_{n}^{4}}{4 v_{0}^{4}}\right)}-\frac{\Delta_{n}^{2}}{2 v_{0}^{2}}\right)^{1 / 2} \\
& +\sum_{n=1}^{N} \frac{1}{2} d_{0} \rho s A \frac{\Delta_{n}^{3}}{T[n]^{2}} \leq E_{\mathrm{tot}} \\
& \\
& \\
& \\
&
\end{aligned}
$$

In (8), when the aggregated data for user $m$ is no less than $Q_{m}, \rho_{m}=1$ and the objective function (7) is increased by 1, otherwise, $\rho_{m}=0$ and (7) remains the same. According to (10), the UAV starts at CBS and finishes at CBS at the end of the trajectory. The UAV mobility is governed by the velocity constraint in (12).

Since (8) has couplings and (15) is nonconvex, problem P1 is difficult to find its globally optimal solution. In the following, we propose an efficient iterative algorithm based on SCA and BCD for addressing P1.

\section{PROPOSED ALGORITHM FOR MAXIMIZING THE NUMBER OF COVERED GUS}

In this section, we propose an efficient iterative algorithm based on BCD to obtain the sub-optimal solution of P1. Define $\mathbf{A}=\left\{\alpha_{m n}, \forall m, n\right\}$ and $\mathbf{Q}=\left\{\mathbf{s}[n], T[n], \forall n, t_{m n}, \forall m, n\right\}$ as the bandwidth allocation set and the UAV trajectory set respectively. We decompose $\mathrm{P} 1$ into two sub-problems and alternately optimize them within each iteration.

\section{A. Bandwidth Allocation with Given Trajectory}

Firstly, with a given UAV trajectory set $\mathbf{Q}$, sub-problem P1.1 allocates bandwidth for users in each path slot

$$
\begin{aligned}
&(\mathrm{P} 1.1) \max _{\left\{\mathbf{A}, \rho_{m}\right\}} \sum_{m=1}^{M} \rho_{m} \\
& \text { s.t. (8), (9) and (14) }
\end{aligned}
$$

Note that the left-hand-side (LHS) of (8) is concave with respect to $\alpha_{m n}$, and other constraints are convex. Thus, P1.1 can be solved via standard convex optimization solvers.

\section{B. Trajectory Optimization with Given Bandwidth Allocation}

Similarly, with a given bandwidth allocation $\mathbf{A}$, UAV trajectory related variables $\mathbf{Q}$ can be optimized by solving the following sub-problem P1.2

$$
\begin{aligned}
(\mathrm{P} 1.2) & : \max _{\left\{\mathbf{Q}, \rho_{m}\right\}} \sum_{m=1}^{M} \rho_{m} \\
\text { s.t. } & (8)-(13),(15)
\end{aligned}
$$

It can be seen that there are couplings between $t_{m n}$ and $\mathbf{s}[n]$ in constraint (8) with given $\alpha_{m n}$. Thus, we introduce variables $f_{m}[n]^{2}$ with

$$
f_{m}[n]^{2}=\alpha_{m n} t_{m n} B \log _{2}\left(1+\frac{\gamma_{m}[n]}{\alpha_{m n}}\right)
$$

and reformulate constraint (8) as,

$$
\sum_{n=1}^{N} f_{m}[n]^{2} \geq \rho_{m} Q_{m}, \forall m
$$

We replace equality (16) with the inequality

$$
\frac{f_{m}[n]^{2}}{t_{m n}} \leq \alpha_{m n} B \log _{2}\left(1+\frac{\gamma_{m}[n]}{\alpha_{m n}}\right)
$$

We observe that the Right-hand-side (RHS) of (18), which is $R_{m}[n]$, is convex with respect to $\left\|\mathbf{s}[n]-\mathbf{w}_{m}\right\|^{2}$. Since any convex function is globally lower-bounded by its first order Taylor expansion at any point [21], SCA can be applied. To be specific, with a local UAV location $\mathbf{s}^{(l)}[n]$ given by $l$-th iteration, we yield the following lower bound $R_{m}^{\mathrm{lb}}[n]$ for $R_{m}[n]$

$$
\begin{aligned}
R_{m}[n] & \geq A_{m}^{(l)}[n] \alpha_{m n} B \\
& -B_{m}^{(l)}[n] \alpha_{m n} B\left(\left\|\mathbf{s}[n]-\mathbf{w}_{m}\right\|^{2}-\left\|\mathbf{s}^{(l)}[n]-\mathbf{w}_{m}\right\|^{2}\right) \\
& \triangleq R_{m}^{\mathrm{lb}}[n]
\end{aligned}
$$

with a constant

$$
A_{m}^{(l)}[n]=\log _{2}\left(1+\frac{P \zeta_{0}}{\alpha_{m n}\left(H^{2}+\left\|\mathbf{s}^{(l)}[n]-\mathbf{w}_{m}\right\|^{2}\right)}\right)
$$


and another constant

$$
\begin{aligned}
B_{m}^{(l)}[n]= & \frac{\left(\log _{2} e\right) P \zeta_{0} / \alpha_{m n}}{\left(H^{2}+\left\|\mathbf{s}^{(l)}[n]-\mathbf{w}_{m}\right\|^{2}\right)} \\
& \times \frac{1}{\left(H^{2}+\left\|\mathbf{s}^{(l)}[n]-\mathbf{w}_{m}\right\|^{2}+\frac{P \zeta_{0}}{\alpha_{m n}}\right)}
\end{aligned}
$$

The equality of (19) holds at the point $\mathbf{s}[n]=\mathbf{s}^{(l)}[n]$. With $R_{m}^{\mathrm{lb}}[n]$, the nonconvex constraint (18) is reformulated as

$$
R_{m}^{\mathrm{lb}}[n] \geq \frac{f_{m}[n]^{2}}{t_{m n}}
$$

For (22), LHS is concave and RHS is convex, it is convex now.

Now, we use (17) and (22) to replace (8). But (17) is still nonconvex. Similarly, the constraint (17) can be handled by applying SCA. Specifically, since the LHS of (17) is convex with respect to $f_{m}[n]$, a lower-bound of $f_{m}[n]^{2}$ can be obtained with any given local point $f_{m}^{(l)}[n]$ as follows

$$
\begin{aligned}
f_{m}[n]^{2} & \geq f_{m}^{(l)}[n]^{2}+2 f_{m}^{(l)}[n]\left(f_{m}[n]-f_{m}^{(l)}[n]\right) \\
& \triangleq \psi_{\mathrm{lb}}\left(f_{m}[n]\right)
\end{aligned}
$$

where the equality holds at the point $f_{m}[n]=f_{m}^{(l)}[n]$. Therefore, the constraint (17) can be written as

$$
\sum_{n=1}^{N} \psi_{\mathrm{lb}}\left(f_{m}[n]\right) \geq \rho_{m} Q_{m}, \forall m
$$

Thus, we reformulate the nonconvex (8) with (22) and (24).

Then, we deal with the nonconvex function (15). Note that the second term of LHS of (15) is nonconvex. We introduce new variables $y_{n}$ with

$$
y_{n}^{2}=\left(\sqrt{\left(T[n]^{4}+\frac{\Delta_{n}^{4}}{4 v_{0}^{4}}\right)}-\frac{\Delta_{n}^{2}}{2 v_{0}^{2}}\right)^{1 / 2}
$$

which is equivalent to

$$
\frac{T[n]^{4}}{y_{n}^{2}}=y_{n}^{2}+\frac{\Delta_{n}^{2}}{v_{0}^{2}}
$$

Then, (15) is written as

$$
\sum_{n=1}^{N}\left[P_{0}\left(T[n]+\frac{\frac{3 \Delta_{n}^{2}}{U_{\mathrm{tip}}^{2}}}{T[n]}\right)+P_{\mathrm{i}} y_{n}+\frac{1}{2} d_{0} \rho s A \frac{\Delta_{n}^{3}}{T[n]^{2}}\right] \leq E_{\mathrm{tot}}
$$

We replace (26) with the inequality as

$$
\frac{T[n]^{4}}{y_{n}^{2}} \leq y_{n}^{2}+\frac{\Delta_{n}^{2}}{v_{0}^{2}}
$$

A lower-bound of RHS of (28) can be obtained as

$$
\begin{aligned}
\frac{T[n]^{4}}{y_{n}^{2}} & \leq y_{n}^{(l) 2}+2 y_{n}^{(l)}\left(y_{n}-y_{n}^{(l)}\right)-\frac{\left\|\mathbf{s}^{(l)}[n+1]-\mathbf{s}^{(l)}[n]\right\|^{2}}{v_{0}^{2}} \\
& +\frac{2}{v_{0}^{2}}\left(\mathbf{s}^{(l)}[n+1]-\mathbf{s}^{(l)}[n]\right)(\mathbf{s}[n+1]-\mathbf{s}[n])^{T} \\
& \triangleq \phi_{\mathrm{lb}}\left(y_{n}, \mathbf{s}[n]\right)
\end{aligned}
$$

where $y_{n}^{(l)}$ is the value of $y_{n}$ in $l$-th iteration.

If any constraint in (18) is satisfied with strict inequality, then we may reduce variable $\gamma_{m}[n]$ to enforce strict equality. Therefore, at the optimal solution to P1.2, all constraints in (18) can be satisfied with equality, likewise for $y_{n}$ and $\mathbf{s}[n]$ in (28). Thus, P1.2 replaced (16) and (26) with (18) and (28) is equivalent with the original P1.2. By replacing constraints (8) and (15) with their lower bounds in each iteration, we have the reformulation as follows

$$
\begin{aligned}
\left(\mathrm{P} 1.2^{\prime}\right): & \max _{\left\{\mathbf{s}[n], f_{m}[n], y_{n}, T[n], t_{m n}, \rho_{m}\right\}} \sum_{m=1}^{M} \rho_{m} \\
\text { s.t. } & \sum_{n=1}^{N} \psi_{\mathrm{lb}}\left(f_{m}[n]\right) \geq \rho_{m} Q_{m}, \forall m, \\
& R_{m}^{\mathrm{lb}}[n] \geq \frac{f_{m}[n]^{2}}{t_{m n}}, \forall n, m, \\
& \frac{T[n]^{4}}{y_{n}^{2}} \leq \phi_{\mathrm{lb}}\left(y_{n}, \mathbf{s}[n]\right), \forall n \\
& (9)-(13) \text { and }(27)
\end{aligned}
$$

Problem P1.2' is a convex optimization problem which can again be solved by standard optimization solvers. Note that due to the global lower bounds in (22), (24) and (29), if the constraints of problem $\mathrm{P} 1.2^{\prime}$ are satisfied, these constraints in the original problem P1.2 are satisfied as well.

\section{Iterative Optimization and Guaranteed Convergence}

We propose an iterative algorithm by solving the two subproblems $\mathrm{P} 1.1$ and $\mathrm{P} 1.2^{\prime}$ based on $\mathrm{BCD}$ for $\mathrm{P} 1$, which is summarized in Algorithm 1. In the following, we prove the convergence of Algorithm 1. Define $\eta\left(\mathbf{A}_{l}, \mathbf{Q}_{l}\right)$ and $\eta_{\mathrm{trj}}^{\mathrm{lb}}\left(\mathbf{A}_{l}, \mathbf{Q}_{l}\right)$ as the objective value of $\mathrm{P} 1$ and $\mathrm{P} 1.2^{\prime}$ respectively. It then follows that

$$
\begin{aligned}
\eta\left(\mathrm{A}_{l}, \mathbf{Q}_{l}\right) & \stackrel{a}{\leq} \eta\left(\mathrm{A}_{l+1}, \mathbf{Q}_{l}\right) \\
& \stackrel{b}{=} \eta_{\mathrm{trj}}^{\mathrm{lb}}\left(\mathrm{A}_{l+1}, \mathbf{Q}_{l}\right) \\
& \stackrel{c}{\leq} \eta_{\mathrm{trj}}^{\mathrm{lb}}\left(\mathrm{A}_{l+1}, \mathbf{Q}_{l+1}\right) \\
& \stackrel{d}{\leq} \eta\left(\mathrm{A}_{l+1}, \mathbf{Q}_{l+1}\right)
\end{aligned}
$$

\footnotetext{
Algorithm 1 Block coordinate descent technique for addressing P1

Initialization: Let $l=0$; initialize the trajectory set $\left\{\mathbf{s}^{(0)}[n], T^{(0)}[n], y_{n}^{(0)}, \forall n, f_{m}^{(0)}[n], t_{m n}^{(0)}, \forall m, n\right\}$

1: repeat

2: $\quad$ solve problem P1.1 with given $\mathbf{s}^{(l)}[n], T^{(l)}[n]$ and $t_{m n}^{(l)}$, and denote the optimal solution as $\alpha_{m n}^{(l+1)}$;

3: $\quad$ solve problem $\mathrm{P} 1.2^{\prime}$ with given $\alpha_{m n}^{(l+1)}, \mathbf{s}^{(l)}[n], f_{m}^{(l)}[n]$ and $y_{n}^{(l)}$, and denote the optimal solution as $\mathbf{s}^{(l+1)}[n]$, $T^{(l+1)}[n], t_{m n}^{(l+1)}, f_{m}^{(l+1)}[n]$ and $y_{n}^{(l+1)}$;

4: $\quad$ update $l=l+1$.

5: until the objective value keeps the same as the value obtained in the previous iteration
} 
where (a) holds since in step 2 of Algorithm 1, the optimal solution $\mathbf{A}_{l+1}$ of P1.1, is obtained based on given $\mathbf{Q}_{l}$; (b) holds since the first order Taylor expansions in (22), (24) and (29) are tight at the given variable, $\mathrm{P} 1.2$ and $\mathrm{P} 1.2^{\prime}$ have the identical objective value; (c) holds due to the fact that with the given $\mathbf{A}_{l+1}$ and $\mathbf{Q}_{l}, \mathrm{P} 1.2^{\prime}$ is optimally solved in step 3 with solution $\mathbf{Q}_{l+1}$; (d) holds as the objective value obtained by solving P $1.2^{\prime}$ serves as the lower-bound of that of the original problem P1.2 at $\mathbf{Q}_{l+1}$. Therefore, (30) suggests that the proposed algorithm is nondecreasing. Since the objective value of P1 is upperbounded by a finite integer value, the algorithm is guaranteed to converge.

\section{INITIAL TRAJECTORY DESIGN}

According to [17], [22], both the converged solution and the performance of such iterative algorithm depend on the initialization schemes. Thus, we focus on a simple circular initial trajectory to initialize sets $\mathbf{A}$ and $\mathbf{Q}$. Since the dispatched UAV has to return to CBS within a time period, the typical initial trajectory for such scenario is a circular trajectory with $T_{\text {tot }}$ [12], [14]. Specifically, for the square target area, we assume that

TABLE II

SIMULATION PARAMETERS

\begin{tabular}{|c|c|c|c|}
\hline parameter & value & parameter & value \\
\hline$B$ & $10^{7} \mathrm{~Hz}$ & $H$ & $100 \mathrm{~m}$ \\
\hline$P$ & $0.01 \mathrm{~W}$ & $\Delta_{\max }$ & $30 \mathrm{~m}$ \\
\hline$\beta_{0}$ & $-30 \mathrm{~dB}$ & $\sigma^{2}$ & $-110 \mathrm{dBm} / \mathrm{Hz}$ \\
\hline$v_{\max }$ & $30 \mathrm{~m} / \mathrm{s}$ & & \\
\hline
\end{tabular}

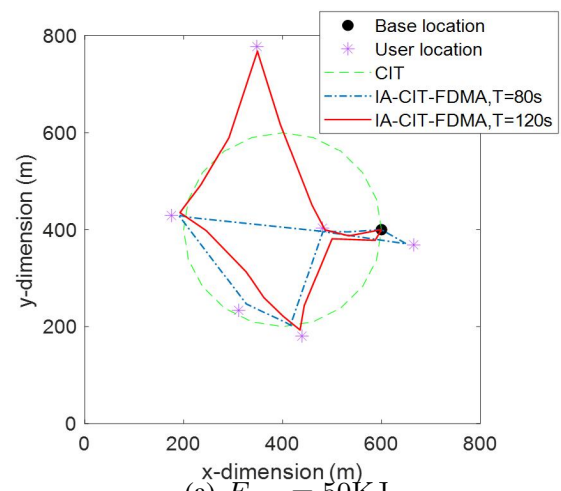

(a) $E_{\text {tot }}=50 \mathrm{KJ}$

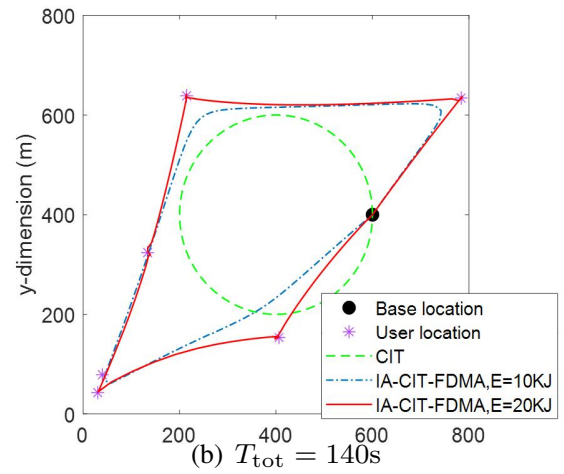

Fig. 2. Optimized trajectories with various time and energy constraints the center of initial trajectory is $\mathbf{c}_{\mathrm{t}}=\left[0.5 L_{s}, 0.5 L_{s}\right] \in \mathbb{R}^{1 \times 2}$ and the radius is $r_{\mathrm{t}}=0.25 L_{s}$, so the number of users inside and outside the trajectory is balanced. For simplicity, we set CBS of both the initial trajectory and our proposed trajectory as $\mathbf{s}_{0}=\mathbf{c}_{\mathrm{t}}+\left[r_{\mathrm{t}}, 0\right]=\left[0.5 L_{s}+r_{\mathrm{t}}, 0.5 L_{s}\right]$.

\section{SIMULATION RESULTS AND ANALYSIS}

In this section, we provide numerical results to evaluate the performance of our proposed techniques. We assume that $M=$ 6 GUs are distributed randomly within the square target area. Unless otherwise stated, we use the parameters shown in Table II. In addition, the data demand of each user is a random value within the range of $[200,500]$ kbits. The coverage performance is evaluated with regard to user coverage probability, which is defined as a ratio of the number of covered users to the number of total users. With a set of given users, increased coverage probability is obtained by increasing the number of covered user.

We apply the circular initial trajectory (CIT) as our benchmark scheme. We exploit two resource allocation schemes for CIT. Firstly, we use time division multiple access (TDMA). In each path slot, each user receives the data with an equal time $T[n] / M$ and total band $B$, and the scheme is termed as CIT-TDMA. Another scheme is CIT-FDMA. In each path slot, the total band $B$ is divided into $M$ equal sub bands, as

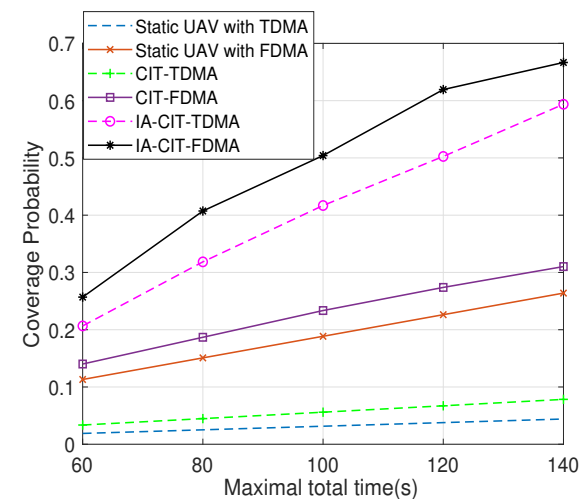

Fig. 3. Coverage probability versus $T_{\text {tot }}$ via different techniques, $E_{\text {tot }}=$ $50 \mathrm{KJ}$

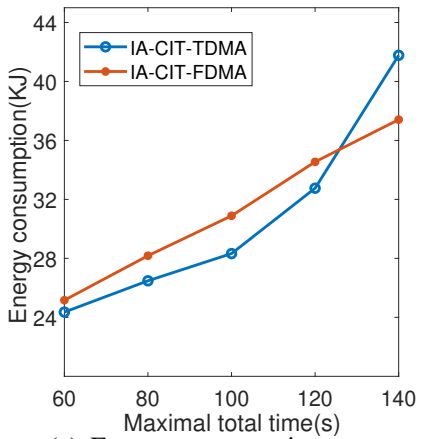

(a) Energy consumption

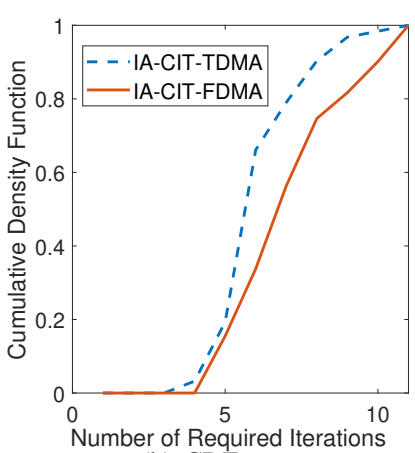

(b) $\mathrm{CDF}$
Fig. 4. Average energy consumption and CDF of IA-CIT-TDMA and IA-CITFDMA, $E_{\mathrm{tot}}=50 \mathrm{KJ}$ 
$\alpha_{m n}=1 / M$ and all users receive the data within the total slot time $T[n]$. And we also deploy a UAV trajectory obtained by the proposed algorithm but without bandwidth allocation termed as IA-CIT-TDMA, and compare IA-CIT-TDMA with our proposed algorithm termed as IA-CIT-FDMA. Specifically, in IA-CIT-TDMA, $t_{m n}$ is optimized via the proposed algorithm with the constraint

$$
\sum_{m=1}^{M} t_{m n} \leq T[n], \forall n
$$

and each user can communicate with the total band $B$.

In Fig. 2, we illustrate IA-CIT-FDMA with various time and energy constraints. Firstly, with a larger $T_{\text {tot }}$, more users data request can be satisfied because more time can be allocated for communication. On the other hand, with the same $T_{\text {tot }}$, more users are covered by increasing $E_{\text {tot }}$ since the UAV can move closer to users to enjoy a better communication condition.

Fig. 3 compares coverage probability with different schemes. We set that the target area here is $1.5 \times 1.5 \mathrm{~km}^{2}$ to obtain a wider coverage increase via time increases. It can be concluded firstly that by exploiting the proposed technique, a better coverage performance is achieved thanks to the reduced communication path loss compared with circular trajectory. Meanwhile, the UAV flying path considering FDMA achieves a higher coverage probability than only uses TDMA.

Fig. 4 further compares the average energy consumption and cumulative distribution function (CDF) for IA-CIT-TDMA and IA-CIT-FDMA. It can be observed that IA-CIT-FDMA consumes more energy than IA-CIT-TDMA when we have a smaller $T_{\text {tot }}$. However, the energy consumption of IA-CITTDMA increases more drastically with $T_{\text {tot }}$ increases. Nevertheless, the trajectory design with bandwidth allocation results in more iteration times compared with the algorithm only based on TDMA. Therefore, in real scenarios, the choice of trajectory design should take both coverage performance and processing time into account.

\section{CONCLUSION}

In this paper, an aerial base station based on rotary-wing UAV is dispatched for covering a maximum number of users with a limited flight time and energy budget. An iterative algorithm alternately optimizes the UAV trajectory and bandwidth allocation based on successive convex approximation and block coordinate descent is proposed. Numerical results verify that the performance can be improved compared with benchmarks.

\section{REFERENCES}

[1] M. Mozaffari, W. Saad, M. Bennis, Y. Nam, and M. Debbah, "A Tutorial on UAVs for Wireless Networks: Applications, Challenges, and Open Problems", CoRR, vol. abs/1803.00680, 2018. [Online]. Available: http://arxiv.org/abs/1803.00680.

[2] Y. Zeng, R. Zhang, and T. J. Lim, "Wireless Communications with Unmanned Aerial Vehicles: Opportunities and Challenges," IEEE Communications Magazine, vol. 54, no. 5, pp. 36-42, May 2016.

[3] E. Kalantari, I. Bor-Yaliniz, A. Yongacoglu, and H. Yanikomeroglu, "User Association and Bandwidth Allocation for Terrestrial and Aerial Base Stations with Backhaul Considerations," in 2017 IEEE 28th Annual International Symposium on Personal, Indoor, and Mobile Radio Communications (PIMRC), Oct 2017, pp. 1-6.
[4] B. Galkin, J. Kibilda, and L. A. DaSilva, "Deployment of UAV-mounted Access Points According to Spatial User Locations in Two-tier Cellular Networks," in 2016 Wireless Days (WD), March 2016, pp. 1-6.

[5] K. Namuduri, Y. Wan, and M. Gomathisankaran, "Mobile Ad Hoc Networks in the Sky: State of the Art, Opportunities, and Challenges," in Proceedings of the Second ACM MobiHoc Workshop on Airborne Networks and Communications, New York, NY, USA, 2013, pp. 25-28.

[6] A. Al-Hourani, S. Kandeepan, and S. Lardner, "Optimal LAP Altitude for Maximum Coverage," IEEE Wireless Communications Letters, vol. 3, no. 6, pp. 569-572, Dec 2014.

[7] M. Mozaffari, W. Saad, M. Bennis, and M. Debbah, "Drone Small Cells in the Clouds: Design, Deployment and Performance Analysis," in 2015 IEEE Global Communications Conference (GLOBECOM), Dec 2015, pp. $1-6$.

[8] M. Alzenad, A. El-Keyi, F. Lagum, and H. Yanikomeroglu, “3-D Placement of an Unmanned Aerial Vehicle Base Station (UAV-BS) for EnergyEfficient Maximal Coverage," IEEE Wireless Communications Letters, vol. 6, no. 4, pp. 434-437, Aug 2017.

[9] R. I. Bor-Yaliniz, A. El-Keyi, and H. Yanikomeroglu, "Efficient 3D Placement of an Aerial Base Station in Next Generation Cellular Networks," in 2016 IEEE International Conference on Communications (ICC), May 2016, pp. 1-5.

[10] J. Sun and C. Masouros, "Deployment Strategies of Multiple Aerial Bss for User Coverage and Power Efficiency Maximization," IEEE Transactions on Communications, vol. 67, no. 4, pp. 2981-2994, April 2018.

[11] I. Valiulahi and C. Masouros, "Multi-UAV Deployment for Throughput Maximization in the Presence of Co-channel Interference," IEEE Transactions on Internet of Things, in press.

[12] J. Lyu, Y. Zeng, and R. Zhang, "UAV-Aided Offloading for Cellula rHotspot," IEEE Transactions on Wireless Communications, vol. 17, no. 6, pp. 3988-4001, June 2018.

[13] Q. Wu and R. Zhang, "Delay-constrained Throughput Maximization in UAV-enabled OFDM Systems," in 2017 23rd Asia-Pacific Conference on Communications (APCC), Dec 2017, pp. 1-6.

[14] Q. Wu, Y. Zeng, and R. Zhang, "Joint Trajectory and Communication Design for Multi-UAV Enabled Wireless Networks," IEEE Transactions on Wireless Communications, vol. 17, no. 3, pp. 2109-2121, March 2018.

[15] X. Jing, J. Sun and C. Masouros, "Energy Aware Trajectory Optimization for Aerial Base Stations," IEEE Transactions on Communications, in press.

[16] M. Hua, Y. Wang, Z. Zhang, C. Li, Y. Huang, and L. Yang, "PowerEfficient Communication in UAV-Aided Wireless Sensor Networks," IEEE Communications Letters, vol. 22, no. 6, pp. 1264-1267, June 2018.

[17] J. Zhang, Y. Zeng, and R. Zhang, "UAV-enabled Radio Access Network: Multi-mode Communication and Trajectory Design," IEEE Transactions on Signal Processing, vol. 66, no. 20, pp. 5269-5284, Oct 2018.

[18] F. Wu, D. Yang, L. Xiao, and L. Cuthbert, "Energy Consumption and Completion Time Tradeoff in Rotary-wing UAV Enabled WPCN," IEEE Access, vol. 7, pp. 79 617-79 635, June 2019

[19] J. Lyu, Y. Zeng, R. Zhang, and T. J. Lim, "Placement Optimization of UAV-Mounted Mobile Base Stations," IEEE Communications Letters, vol. 21, no. 3, pp. 604-607, March 2017.

[20] Y. Zeng, R. Zhang, and T. J. Lim, "Throughput Maximization for UAVEnabled Mobile Relaying Systems," IEEE Transactions on Communications, vol. 64, no. 12, pp. 4983-4996, Dec 2016.

[21] S. Boyd and L. Vandenberghe, Convex Optimization. New York, NY, USA: Cambridge University Press, 2004.

[22] M. Hong, M. Razaviyayn, Z. Luo, and J. Pang, "A Unified Algorithmic Framework for Block-Structured Optimization Involving Big Data: With Applications in Machine Learning and Signal Processing," IEEE Signal Processing Magazine, vol. 33, no. 1, pp. 57-77, Jan 2016. 